\title{
La Libertad Vigilada como medida individualizadora en la Justicia de Menores.
}

\author{
María José Bernuz Beneitez. Universidad de Zaragoza. \\ Esther Fernández Molina. Universidad de Castilla La Mancha. \\ Fátima Pérez Jiménez. Universidad de Málaga.
}

\begin{abstract}
RESUMEN
La medida de Libertad Vigilada es un clásico entre los recursos de medio abierto con los que ha contado el Juez de menores a lo largo de la historia de la justicia de menores; fundamentalmente, porque en teoría su contenido educativo y la supervisión que realiza el educador, permite una mejor adaptación de la intervención a las circunstancias y a la evolución del menor. En este trabajo se presentan los resultados de una investigación empírica realizada de manera comparada en tres provincias españolas, Zaragoza, Málaga y Toledo, en la que se ha tratado de conocer con detenimiento cómo se ejecuta esta medida, para conocer mejor cómo se concreta en la práctica. En concreto, uno de los principales objetivos que se plantea este trabajo es analizar si realmente la medida de Libertad Vigilada consigue individualizar la respuesta que se proporciona a los menores infractores, tanto a las circunstancias psicosociales del menor cuando éste comete el delito, como a la evolución del mismo a lo largo de la ejecución de la medida. Los resultados de la investigación sugieren que a pesar de la respuesta especial y distinta que se da en la justicia de menores que pretende valorar las circunstancias psicosociales del menor, se aprecia cierta homogeneidad en las intervenciones.
\end{abstract}

Palabras clave: justicia de menores, libertad vigilada, alternativas al internamiento, intervención, individualización.

Revista Española de Investigación Criminológica

Artículo 6, Número 7 (2009) $\quad$ www.criminología.net

ISSN: $1696-9219$ 


\begin{abstract}
Along the story of juvenile justice, probation has been a common feature amongst the open-institutional measures on which the Juvenile Judge has relied on; basically because in theory, its educational contents and the advisory role of the probation officer allows for a better adjustment of the legal intervention to the youth's circumstances and evolution. In the present study we report the results of an empirical research conducted simultaneously in three Spanish provinces, namely Zaragoza, Malaga and Toledo. The overall objective of the study was to grasp in detail how parole is applied in order to better understand how it settles in practice. One of the principal aims of this study is to analyze if the adoption of probation succeeds in individualizing the response given to juvenile offenders, that is, to the psycho-social circumstances (at the time the juvenile commits the crime) and to the evolution to which the juvenile is subjected throughout the enforcement process. The results of this investigation suggest that in spite of the alleged special responses to juvenile offenders - which in principle should take into account the particular circumstances of the juveniles --, we observe the existence of certain homogeneity conditions about the interventions.
\end{abstract}

Key words: Juvenile justice, probation, open-institutional measures, intervention with juveniles, individualising probation

\title{
1. Planteamiento actual del problema: la Libertad Vigilada en la justicia de menores
}

Son muchas las voces que desde los años 90 se han proyectado sobre la justicia de menores y la han calificado como una jurisdicción especializada, tanto por los sujetos con los que se interviene -los menores de entre 14 y 18 años que han cometido un delito o falta - como por los profesionales implicados en ella. En este sentido, es evidente que la necesidad de tener en cuenta el interés del menor en todas las decisiones que les puedan afectar, exige contar con un equipo de operadores jurídicos y también sociales que colaboren en la toma de decisiones interdisciplinares y en la ejecución de las medidas. Otra de las características que la hacen especial es la necesidad impuesta por la ley, pero también consensuada a nivel social, de que las decisiones que se tomen deben ser individualizadas; esto es, deben tener en cuenta el delito cometido y las circunstancias en que se cometieron, pero sobre todo deben asumir las circunstancias de los menores (sociales, educativas, familiares,...) para adaptar la medida a sus trayectorias, capacidades y posibilidades. Un paso imprescindible para ello es que las Comunidades Autónomas provean al juez de todas las medidas previstas legalmente para que sea la medida la que se ajuste a las necesidades del menor. Igualmente, tanto el

Revista Española de Investigación Criminológica

Artículo 6, Número 7 (2009) $\quad$ www.criminología.net

ISSN: $1696-9219$ 
procedimiento como los profesionales deberán favorecer y promover la revisión de las medidas para adaptarlas a la evolución efectiva del menor.

La medida de Libertad Vigilada y su ejecución nos permiten analizar todos estos elementos e indagar el grado real de individualización de la justicia de menores. Hay que indicar que la Libertad Vigilada ha sido una medida tradicional lo largo de la historia de la justicia de menores española, y sigue ocupando hoy en día un lugar clave en ese amplio abanico de medidas propuestas por la LO 5/2000. De hecho, la propia normativa -que ordena las medidas en función de la restricción de derechos que suponen - la considera como la medida más dura y exigente después del internamiento del tipo que sea. Ya destacábamos en un trabajo anterior dedicado a la ejecución de esta medida (Bernuz, Fernández, Pérez, 2009) que "se trata de una medida que permite al menor seguir en un régimen de libertad aunque supervisada por un educador del Equipo de Medio Abierto que controla que el menor realice $-\mathrm{O}$ no- las tareas encomendadas por el juez a propuesta del educador y a la vista del informe psicosocial del menor, así como del tipo y las circunstancias de la comisión del delito. De este modo, se responde a ese interés del menor que exige tanto su sanción - por el delito cometido- como su educación -a través de la medida". Por todo ello, en este trabajo, se va a analizar la Libertad Vigilada como la medida que por su contenido y supervisión por un educador, permite una mejor adaptación de la intervención a las circunstancias y a la evolución del menor.

\section{Objetivos de trabajo}

Con este planteamiento inicial de trabajo, y con el conocimiento de la ejecución de la medida de Libertad Vigilada que obtuvimos al realizar un estudio previo, son varios los objetivos que se persiguen con este trabajo. El objetivo fundamental del estudio es analizar si realmente la medida de Libertad Vigilada consigue individualizar la justicia de menores adaptándose, a través de las actividades propuestas, tanto a las circunstancias psicosociales del menor cuando éste comete el delito, como a la evolución del mismo a lo largo de la ejecución de la medida. Al tiempo que se tratará de analizar los factores que pueden incidir en esta realidad: sea el abanico de recursos disponibles, el respeto de las garantías procedimentales, los mecanismos de 
coordinación entre operadores jurídicos y sociales, o el propio régimen de ejecución de las medidas.

Parece claro que para conseguirlo, otro de los objetivos es analizar el tipo de población a la que va dirigida la medida, cuáles son efectivamente sus circunstancias psico-sociales y sus necesidades educativas y de control. Si se parte de que se trata de la medida comunitaria más gravosa y con mayor nivel de control, antes de pasar al internamiento, podemos presuponer que irá dirigida fundamentalmente a menores que han cometido delitos con una cierta gravedad y, sobre todo, que cuentan con una problemática familiar y social importante.

\section{Metodología}

Para conocer el contenido y desarrollo de la ejecución de la Libertad Vigilada bajo el régimen que prevé la LO 5/2000, se proyectó un estudio exploratorio de carácter empírico en tres Comunidades Autónomas: Andalucía, Aragón y Castilla-La Mancha.

\subsection{Muestra}

Se han analizado en profundidad 20 expedientes por Comunidad Autónoma ${ }^{1}$ que contuvieran las tres modalidades de Libertad Vigilada (todas, excepto como medida cautelar): como medida definitiva (artículo 7.1.h), como medida consecuencia de una suspensión del internamiento en centro cerrado (artículo 40.2), o como medida tendente a favorecer la desinstitucionalización mediante el desdoblamiento de la medida de internamiento (artículo 7.2). En concreto, se examinaron los 15 primeros expedientes que se incoaron por los servicios de ejecución de medidas con una Libertad Vigilada como medida definitiva impuesta por el Juez de menores, en el año 2006. Así como los cinco primeros expedientes que durante ese año obtuvieron una medida de Libertad Vigilada en las otras dos modalidades. De este modo la muestra total la componen 60 expedientes.

\footnotetext{
${ }^{1}$ Para conocer la realidad de la Comunidad Autónoma de Andalucía se analizaron expedientes en Málaga, en Aragón la provincia de donde proceden los expedientes es Zaragoza y Toledo para Castilla-La Mancha. En Zaragoza, en el año 2006, se impusieron 255 medidas de libertad vigilada. En Málaga, en ese mismo año, las medidas de libertad vigilada impuestas fueron 864 y en Toledo fueron 199.
}

Revista Española de Investigación Criminológica 


\subsection{Cuestionario y variables utilizadas}

Para facilitar la recogida de la información que se precisaba para la investigación se elaboró un cuestionario ad hoc que se apoyó en los documentos de trabajo que la comunidad autónoma de Castilla-La Mancha ha elaborado para diseñar el Programa Individualizado de Ejecución de Medida (en adelante PIEM) (Bernuz, Fernández, Pérez, 2009).

Así pues, el cuestionario consta de varias partes. En un primer bloque se incluyen las variables que recogen toda la información relativa al menor y al expediente. Al inicio constan los datos que permiten identificar el expediente: número, fecha y lugar de nacimiento del menor, sexo, lugar de residencia, delito cometido y fecha de comisión del mismo. Junto a ello, están las variables que dan a conocer los antecedentes del menor en Fiscalía y en el Juzgado, identificando el tipo de delito cometido y la medida impuesta, para así poder conocer el historial de intervención que ha tenido cada menor.

También en este primer bloque de variables se reune toda la información psicosocial sobre el menor disponible en el informe del Equipo técnico y en el del técnico de Libertad Vigilada. Así, en relación con las variables familiares se ha recogido información de los siguientes aspectos: estructura de la familia, dinámica en las relaciones entre los distintos miembros, valorando, en su caso, la existencia de maltrato familiar y/o abuso sexual, problemática de los progenitores y contacto con los servicios sociales. Con toda esta información finalmente, se ha diseñado una variable que la agrupa y que clasifica a los menores en cuatro categorías: como menores sin problemática familiar cuando no presentan problemas en ninguno de los aspectos citados, problemática familiar leve cuando sólo presentan uno o dos problemas, problemática moderada cuando presentan al menos tres problemas familiares $\mathrm{y}$ problemática familiar grave cuando presentan cuatro o cinco problemas familiares ${ }^{2}$.

\footnotetext{
${ }^{2}$ Como puede observarse al hacer esta clasificación se ha adoptado el criterio de no considerar ningún problema familiar más grave que otro. La dificultad a la hora de determinar cómo de graves pueden ser estos problemas en la esfera familiar y la diferente intensidad con la que se pueden producir y pueden afectar individualmente a cada menor, nos ha llevado a definir la gravedad de la conducta familiar por el número de problemas que concurren.
}

Revista Española de Investigación Criminológica

Artículo 6, Número 7 (2009) $\quad$ www.criminología.net

ISSN: $1696-9219$ 
En relación con las variables relativas a la situación formativo/laboral del menor se ha indagado sobre su situación escolar midiendo si el menor está o no escolarizado y en este último caso, si está trabajando. También se ha recogido información en todos los supuestos de cómo es o fue su paso por la escuela, intentando identificar problemas de absentismo, de conducta y de rendimiento. Ello ha dado lugar a una variable que engloba toda la información escolar que cuenta con los siguientes niveles de análisis: sin problemática escolar, con problemática leve cuando cuenta con cualquiera de los tres problemas citados, moderada cuando cuenta con dos y grave cuando cuenta con los tres problemas.

Para valorar el estilo de vida de estos jóvenes se ha identificado el tipo de grupo de pares con el que se relaciona, si son o no conflictivos y las conductas de riesgo que realizan: consumos de alcohol y drogas, fugas de casa, promiscuidad sexual y comportamientos violentos.

Se ha intentado también determinar la salud física y mental de los menores, identificando si hubiera problemas en cualquiera de estos aspectos y en el último caso, si existe un diagnóstico por parte de los servicios de Salud mental.

Un segundo bloque de variables estaría formado por aquéllas que hacen referencia a la tramitación del expediente: constancia de medida cautelar, ejercicio de acusación particular, conformidad o no con la medida, medidas propuestas por el Fiscal, el letrado de la defensa y el Equipo técnico, existencia y condiciones de suspensión o de sustitución de la medida.

El tercer bloque de variables hace referencia al desarrollo de la ejecución de la medida. Desde la tramitación procesal que supone la misma, en donde se han recogido todas las fechas en las que se han ido realizando los distintos trámites, hasta el diseño del PIEM. En este caso, se han identificado la periodicidad de las entrevistas y las actividades propuestas, los objetivos, las actividades concretas que el menor debe realizar y los recursos con los que se ha dispuesto.

Un cuarto bloque de variables ha tratado de medir los resultados obtenidos con la ejecución de la medida. Así, se ha recogido información sobre el grado de asistencia a 
las entrevistas y a las actividades propuestas, midiéndolas en tres niveles de análisis: asistencia habitual (más del 75\%), asistencia media (entre el 75\% y el 50\%) y asistencia deficiente (menos del 50\%). También se ha recogido información sobre el cumplimiento del plan de trabajo que podía ser: siempre, casi siempre, a veces y nunca. Otro de los aspectos que se ha intentado valorar es el grado de cumplimiento de los objetivos y la implicación del menor, para ello se ha utilizado la valoración personal que los técnicos han realizado sobre el grado de consecución de objetivos (consecución positiva, media, deficiente o nula) y sobre la implicación del menor (buena, regular, escasa o nula).

Así mismo, se han valorado también los cambios que el desarrollo de la ejecución de la medida ha provocado en el menor. En atención a las cinco grandes áreas de intervención que propone el artículo 18 del R.D. 1774/2004 (responsabilización, familiar, formativo/laboral, ocio y personal) se ha identificado si el menor ha obtenido cambios en positivo, si no ha cambiado o lo ha hecho negativamente y si no había necesidad de cambio en algún área en particular.

Finalmente, se ha tratado de identificar un posible pronóstico del menor. En este caso, esta variable hace referencia a la valoración que los profesionales que han ejecutado la medida realizan sobre cuál es su impresión sobre el desarrollo de la misma y su posible pronóstico (muy favorable, favorable, regular, estancado o desfavorable). Por otra parte, también se han registrado aquellos casos en los que ha habido un incumplimiento de la medida, decretado formalmente por el Juez de menores y aquellos que han reincidido con posterioridad a la ejecución de la medida.

\subsection{Trabajo de campo}

Para obtener la información y cumplimentar los cuestionarios se acudió a los expedientes archivados en los Equipos de Medio Abierto de Málaga, Zaragoza y Toledo. Asimismo se procedió a completar la información de los expedientes con entrevistas semiabiertas a los profesionales que hicieron el seguimiento de las medidas de Libertad Vigilada analizadas. 


\section{Los resultados}

\subsection{Características de la muestra}

Como ya se indicó, uno de los objetivos del estudio es analizar la población a la que se impone una medida de Libertad Vigilada. Se trata de considerar no sólo su perfil psicosocial y el historial delictivo, sino que también es preciso dar cuenta de su edad, género y nacionalidad. De entrada, hay que indicar que, quizás por tratarse de una medida tradicional en la justicia de menores y que representa un porcentaje importante de la actividad de los Equipos de Medio abierto, lo cierto es que la muestra analizada no se aleja demasiado de la que constituye una muestra típica en la justicia de menores ${ }^{3}$.

Así, en cuanto al género, se puede comprobar cómo los datos que se han obtenido con la muestra se ajustan al perfil con el que se trabaja en la justicia de menores. En efecto, los resultados muestran que el porcentaje de chicas a las que se ha aplicado una medida de Libertad Vigilada es de un $20 \%$, que si bien es algo más alto que los datos que se han obtenido en otros estudios, revelan que el porcentaje de chicas es mucho menor. Por lo general los chicos cometen delitos de robo con fuerza y violencia, mientras las chicas delitos de amenazas, maltrato familiar o robo de uso con violencia (ver tabla 1). En todo caso, es preciso destacar que ningún menor que cumple Libertad Vigilada lo hace por un delito grave, tampoco por una falta. En concreto, el 68,3\% ha cometido un delito de menor gravedad pero con violencia e intimidación y el 31,7\% ha cometido un delito menos grave sin violencia e intimidación.

Si analizamos la edad, también los datos confirman ciertas evidencias que la investigación criminológica ha puesto de manifiesto. Así, se puede observar un aumento lento pero progresivo de la delincuencia en las franjas inferiores de la justicia de menores (ver tabla 1). No obstante, la información más interesante en este sentido, es la que permite conocer el desarrollo de la carrera criminal de estos menores y el momento en el que el sistema proporciona la respuesta. Los resultados del estudio muestran que el

${ }^{3}$ Entre otros, los estudios que informan de estos aspectos son: Bernuz (1999), Rechea y Fernández (2001), Capdevilla, Ferrer y Luque (2005), Pérez (2006), Graña, Garrido y González (2007), García, Díez, Pérez y García (2008), Fernández (2008) y San Juan y Ocáriz 2009).

Revista Española de Investigación Criminológica

Artículo 6, Número 7 (2009) $\quad$ www.criminología.net

ISSN: $1696-9219$ 
primer contacto de los menores de la muestra con la justicia juvenil suele producirse a los 14,65 años (de media), esto es, casi al inicio del ámbito subjetivo de aplicación de la Ley de responsabilidad penal de los menores que está en los 14 años. Por otra parte, la edad media de comisión del delito por el que se les ha impuesto la Libertad Vigilada es a los 15,53 años, mientras que la edad media en la que se inicia la ejecución de esta medida son los 17,34 años.

Finalmente, si se analiza la muestra atendiendo a la nacionalidad, se puede observar que el porcentaje de extranjeros es de un $16,7 \%$, datos que de nuevo coinciden con ese perfil tipo del menor que llega a los Juzgados de menores. No obstante, llama la atención que son los casos más violentos los cometidos por inmigrantes (ver tabla 1).

Tabla 1. Características de la muestra

\begin{tabular}{|l|c|c|c|c|c|c|c|}
\hline & \multicolumn{2}{|c|}{ Sexo } & \multicolumn{2}{c|}{ Edad } & \multicolumn{2}{c|}{ Nacionalidad } & Total \\
\cline { 2 - 8 } & $\begin{array}{c}\text { Hombre } \\
(\%)\end{array}$ & $\begin{array}{c}\text { Mujer } \\
(\%)\end{array}$ & $\begin{array}{c}14 \text { y } 15 \\
\text { años } \\
(\%)\end{array}$ & $\begin{array}{c}16 \text { y } 17 \\
\text { años } \\
(\%)\end{array}$ & $\begin{array}{c}\text { Español } \\
(\%)\end{array}$ & $\begin{array}{c}\text { Extranjero } \\
(\%)\end{array}$ & $(\%)$ \\
\hline $\begin{array}{l}\text { Robo con } \\
\text { violencia }\end{array}$ & 96 & 4 & 36 & 64 & 84 & 16 & 41,7 \\
\hline $\begin{array}{l}\text { Robo con } \\
\text { fuerza }\end{array}$ & 100 & 0 & 44,4 & 55,6 & 77,8 & 22,2 & 15 \\
\hline $\begin{array}{l}\text { Trafico de } \\
\text { drogas }\end{array}$ & 80 & 20 & 20 & 80 & 100 & 0 & 8,3 \\
\hline Lesiones & 75 & 25 & 50 & 50 & 75 & 25 & 6,7 \\
\hline $\begin{array}{l}\text { Amenazas y } \\
\text { coacciones }\end{array}$ & 50 & 50 & 75 & 25 & 75 & 25 & 6,7 \\
\hline $\begin{array}{l}\text { Maltrato } \\
\text { familiar }\end{array}$ & 50 & 50 & 100 & 0 & 75 & 25 & 6,7 \\
\hline $\begin{array}{l}\text { Robo de uso } \\
\text { con violencia }\end{array}$ & 66,7 & 33,3 & 33,3 & 66,7 & 100 & 0 & 5 \\
\hline Hurto & 100 & 0 & 50 & 50 & 100 & 0 & 3,3 \\
\hline $\begin{array}{l}\text { Robo de uso } \\
\text { con fuerza }\end{array}$ & 100 & 0 & 50 & 50 & 50 & 50 & 3,3 \\
\hline $\begin{array}{l}\text { Malos tratos } \\
\text { habituales }\end{array}$ & 100 & 0 & 100 & 0 & 100 & 0 & 1,7 \\
\hline $\begin{array}{l}\text { Allanamiento } \\
\text { morada }\end{array}$ & 100 & 0 & 0 & 100 & 100 & 0 & 1,7 \\
\hline Total & $\mathbf{8 6 , 7}$ & $\mathbf{1 3 , 3}$ & $\mathbf{4 5}$ & $\mathbf{5 5}$ & $\mathbf{8 3 , 3}$ & $\mathbf{1 6 , 7}$ & 100 \\
\hline
\end{tabular}

*Elaboración propia con los datos extraídos de expedientes 


\subsection{Circunstancias psicosociales de los menores que han cumplido Libertad Vigilada}

Las circunstancias psicosociales son, en la justicia de menores, elementos de notable importancia, ya que el juez ha de tomarlas en cuenta a la hora de imponer la medida judicial al menor encausado permitiendo así la individualización de la medida (artículo 7.3). En este apartado se va a exponer con detenimiento cuál es la realidad individual, familiar y social de los menores de la muestra reflejada en los informes realizados por los educadores.

Si se parte de la idea de que las circunstancias familiares en que el menor vive y se desarrolla son las más relevantes de cara a una socialización y crecimiento personal adecuados, se podrían sacar algunas conclusiones cuando se aprecia que un buen número de familias presenta situaciones problemáticas sea en su convivencia, en el estilo educativo, en los problemas de los miembros de la familia que repercuten en el propio entorno familiar, las situaciones conflictivas y de malos tratos o el acceso a Servicios Sociales con anterioridad. De hecho, sólo un $16,7 \%$ de familias viven sin circunstancias familiares distorsionadoras (tabla 2).

Tabla 2. Circunstancias familiares

\begin{tabular}{|l|c|c|}
\hline \multirow{2}{*}{} & \multicolumn{2}{|c|}{ Familia } \\
\cline { 2 - 3 } & $\mathrm{n}$ & $\%$ \\
\hline Carece de problemática & 10 & 16,7 \\
\hline Problemática leve & 26 & 43,3 \\
\hline Problemática moderada & 13 & 21,7 \\
\hline Problemática grave & 11 & 18,3 \\
\hline Total & 60 & 100 \\
\hline
\end{tabular}

De hecho, se podría pensar que uno de los posibles elementos que han influido en la conducta de los hijos es el estilo educativo de estas familias, ya que en el 68,3\% de los casos consta que la dinámica educativa ha sido de alguna forma inadecuada: negligente, autoritaria, permisiva y/o sobreprotectora. Además, en una de cada cuatro familias, el padre o la madre tienen una diversidad de problemas. Entre los hombres el problema más común suele ser la drogadicción, el consumo abusivo de alcohol y el comportamiento delictivo, mientras que las mujeres tienen circunstancias más dispares: 
drogadicción, problemas psicológicos, salud precaria y problemas laborales. En el mismo porcentaje de familias, una de cada cuatro, hay situaciones de malos tratos. Lo que es sorprendente es que la mitad de los casos corresponden a la violencia que ejerce un adulto de la familia hacia el resto, mientras que la otra mitad son maltratos ejercidos por los hijos hacia sus padres. Sólo se constató un caso de abuso sexual de un progenitor a su hija. También es destacable que la mitad de las familias ha acudido en algún momento a los Servicios Sociales. En la mayoría de los casos $(28,3 \%)$ se han solicitado ayudas económicas o de apoyo puntual; pero, en el 18,4\% de las ocasiones la petición de ayuda estaba relacionada con el menor: petición de intervención, de acogimiento o de tutela temporal.

Por último, puesta en relación esta variable de problemática familiar con las otras que reflejan las situaciones difíciles en la vida del menor (con la familia, en el colegio, con amigos problemáticos y/o una salud mental deficiente) se comprueba que la única relación estadísticamente significativa ${ }^{4}$ se producen entre esta variable y el número de conductas de riesgo del menor (tabla 3). Si se observa detenidamente la tabla se puede comprobar que, efectivamente, existe una relación entre la no problemática familiar y la ausencia de conductas de riesgo.

Tabla 3. Relación entre el $\mathbf{n}^{0}$ de conductas de riesgos y problemática familiar

\begin{tabular}{|c|c|c|c|c|c|}
\hline & \multicolumn{4}{|c|}{$\mathrm{N}^{0}$ conductas de riesgo } \\
\hline & & $\begin{array}{ll} & \mathbf{0} \\
\mathrm{n} & (\%)\end{array}$ & $\begin{array}{ll} & \mathbf{1} \\
\mathrm{n} & (\%)\end{array}$ & $\begin{array}{l}\mathbf{2} \\
\mathrm{n}(\%)\end{array}$ & $\begin{array}{c}3 \\
\text { n }(\%)\end{array}$ \\
\hline \multirow{2}{*}{$\begin{array}{l}\text { Problemática } \\
\text { familiar }\end{array}$} & No & $10(43,5)$ & $0 \quad(0)$ & $0 \quad(0)$ & $0 \quad(0)$ \\
\hline & Sí & $13(56,5)$ & $16(100)$ & $14(100)$ & $7(100)$ \\
\hline
\end{tabular}

Igualmente es preciso analizar el estilo de vida de los adolescentes, ya que en esta etapa de la vida, el uso del tiempo escolar y de ocio y los amigos tiene tanta o más importancia que la familia en la consolidación de una socialización adecuada. En la muestra se aprecia que, a pesar de su edad, son muy pocos los jóvenes que tienen una actividad concreta y cotidiana, unas obligaciones mínimas y unos horarios que cumplir. Es muy significativo el hecho de que sólo el 28,8\% de los menores acuda al colegio en

${ }^{4}$ Los resultados de la tabla demuestran que efectivamente hay una relación entre la variable problemática familiar y número conductas de riesgo. Pero dado que el nivel "no" de la variable problemática familiar es una constante, se ha calculado el Chi-cuadradado dicotomizando la variable número de conductas de riesgo para posibilitar la comparación $\left(\chi^{2}=19,304 ; \mathrm{g} .1 .=1 ; \mathrm{p} \leq 0,01\right)$.

Revista Española de Investigación Criminológica

Artículo 6, Número 7 (2009) $\quad$ www.criminología.net

ISSN: $1696-9219$ 
el momento de la ejecución de la Libertad Vigilada y de éstos, el 64,7\% tenga problemas de conducta. A esto se suma la presencia de una diversidad de conductas de riesgo. De hecho, el consumo de drogas es más habitual que el consumo de alcohol. También se aprecia que uno de cada cuatro menores tenía recogido en su expediente la realización de conductas violentas, consistentes en agresiones y amenazas a compañeros de colegio, ausencia de control de impulsos, reacciones sobredimensionadas e incluso violencia verbal y física con su familia. Más de la mitad de los menores, el 63,3\%, se relaciona con otros chicos y chicas considerados 'problemáticos' por ser consumidores de drogas, tener comportamientos violentos y agresivos y/o cometer actos delictivos.

Finalmente, hay que indicar que los problemas de salud mental tampoco son minoritarios entre los menores y además lo son de índole muy diversa: trastornos en el desarrollo cognitivo, trastornos emocionales y comportamientos perturbados. Es importante indicar que de todos ellos, sólo unos pocos $(11,7 \%)$ tienen un diagnóstico clínico de su enfermedad (tabla 4).

Tabla 4. Circunstancias negativas

\begin{tabular}{|l|c|c|}
\hline & n & \% \\
\hline Problemática moderada o grave en colegio & 47 & 78,3 \\
\hline Consumo de droga & 33 & 55 \\
\hline Consumo de alcohol & 19 & 31,7 \\
\hline Problemas de salud mental & 20 & 33,3 \\
\hline Pares problemáticos & 38 & 63,3 \\
\hline
\end{tabular}

\subsection{Historial delictivo y de intervención del menor}

Según los resultados obtenidos en el análisis, el 56,6\% de los menores de la muestra tenían antecedentes en Fiscalía y/o Juzgados de menores, por lo tanto son reincidentes. No obstante, a pesar de ser reincidentes sólo el $41,6 \%$ (de ese 56,6\%) han cumplido ya otras medidas, esto es, no todos los menores que han cometido un delito han obtenido una respuesta judicial. En su mayoría las medidas que han cumplido previamente son otras libertades vigiladas; aunque, en algunos casos, en un $20 \%$ del total, los menores han cumplido previamente una medida de internamiento. Estos resultados ponen de manifiesto, por un lado, que la Libertad Vigilada se aplica a un porcentaje bastante alto de menores $(58,4 \%)$ como primera opción de intervención; esto 
es, sin probar antes con otras medidas de intervención comunitaria más suaves, sobre todo cuando, como se decía con anterioridad, los hechos cometidos no son especialmente graves. Por otro lado, es apreciable que con algunos menores la intervención combina medio abierto y privación de libertad simultáneamente, a juzgar al menos por ese $20 \%$ de menores que aunque ahora estén cumpliendo una medida de Libertad Vigilada, ya saben lo que es estar internados en un centro en cualquiera de los regímenes.

Por otra parte, se ha tratado de delimitar un perfil de estos menores que tienen ya un historial delictivo para distinguirlos de aquéllos que no son reincidentes. De todas las variables analizadas aquellas que presentan diferencias significativas son las que muestra la tabla 5 .

Tabla 5. Características psicosociales de los menores con historial delictivo

\begin{tabular}{|c|c|c|c|c|}
\hline & & $\begin{array}{c}\text { Menor con historial } \\
\text { delictivo } \\
\mathrm{N}(\%)\end{array}$ & $\begin{array}{c}\text { Menor sin historial } \\
\text { delictivo } \\
\mathrm{N}(\%)\end{array}$ & Prueba de significación \\
\hline \multirow{2}{*}{$\begin{array}{l}\text { Problemas } \\
\text { familiares }\end{array}$} & Sí & $33(66)$ & $17(34)$ & \multirow{2}{*}{$\chi^{2}=10,883 ;$ g. $1 .=3 ; p \leq 0,01$} \\
\hline & No & $1(10)$ & $9(90)$ & \\
\hline \multirow{2}{*}{$\begin{array}{l}\text { Amigos } \\
\text { conflictivos }\end{array}$} & Sí & $26(68,4)$ & $12(31,6)$ & \multirow{2}{*}{$\chi^{2}=5,454 ;$ g.1. $=1 ; p<0,05$} \\
\hline & No & $5(33,3)$ & $10(66,7)$ & \\
\hline \multirow{2}{*}{$\begin{array}{l}\text { Consumo de } \\
\text { drogas }\end{array}$} & Sí & $23(69,7)$ & $10(30,3)$ & \multirow{2}{*}{$\chi^{2}=8,266 ;$ g.1. $=1 ; \mathrm{p} \leq 0,01$} \\
\hline & No & $5(27,8)$ & $13(72,2)$ & \\
\hline \multirow{2}{*}{$\begin{array}{l}\text { Consumo de } \\
\text { alcohol }\end{array}$} & Sí & $13(68,4)$ & $6(31,6)$ & \multirow{2}{*}{$\chi^{2}=6,352 ;$ g.1. $=1 ; p \leq 0,01$} \\
\hline & No & $6(28,6)$ & $15(71,4)$ & \\
\hline
\end{tabular}

Si se observa la tabla 5, los sujetos que tienen antecedentes en Fiscalía y/o Juzgados de menores son chicos con problemas familiares (en especial, relacionados con los patrones de crianza y la dinámica familiar), tienen amigos delincuentes y son consumidores de drogas y alcohol en mayor medida que los que no tienen antecedentes. Así mismo la tabla 6 nos permite analizar la incidencia que puede tener la intervención en la vida posterior de los menores. Podemos ver que lo que diferencia los menores que cuentan con historial de intervención respecto a los que no lo tienen, son los problemas en la escuela y los consumos de drogas y alcohol principalmente. De tal forma que los menores que no tienen historial de intervención tienen una situación formativo/laboral más prometedora y tienen menos problemas con los consumos de tóxicos. 
Tabla 6. Características psicosociales de los menores con historial de intervención

\begin{tabular}{|c|c|c|c|c|}
\hline & \multirow{3}{*}{$\begin{array}{c}\text { Menor con historial } \\
\text { de intervención } \\
\mathrm{N}(\%) \\
24(51,1 \%)\end{array}$} & \multirow{3}{*}{$\begin{array}{c}\text { Menor sin historial } \\
\text { de intervención } \\
\mathrm{N}(\%) \\
23(48,9 \%)\end{array}$} & \multirow{3}{*}{$\begin{array}{l}\text { Prueba de significación } \\
\gamma^{2}=7881 \cdot \sigma 1=1 \cdot n<001\end{array}$} \\
\hline & & & & \\
\hline \multirow{2}{*}{$\begin{array}{l}\text { Problemas } \\
\text { escolares }\end{array}$} & Sí & & & \\
\hline & No & $1(7,7 \%)$ & $12(92,3 \%)$ & $\chi^{2}=7,881 ;$ g.1. $=1 ; p \leq 0,01$ \\
\hline \multirow{2}{*}{$\begin{array}{l}\text { Consumo } \\
\text { de drogas }\end{array}$} & Sí & $17(51,5 \%)$ & $16(48,5 \%)$ & \multirow{2}{*}{$\chi^{2}=5,934 ;$ g.l. $=1 ; p \leq 0,01$} \\
\hline & No & $3(16,7 \%)$ & $15(83,3 \%)$ & \\
\hline \multirow{2}{*}{$\begin{array}{l}\text { Consumo } \\
\text { de alcohol }\end{array}$} & Sí & $10(52,6 \%)$ & $9(47,4 \%)$ & \multirow{2}{*}{$\chi^{2}=6,686 ;$ g.1. $=1 ; p \leq 0,01$} \\
\hline & No & $3(14,3 \%)$ & $18(85,7 \%)$ & \\
\hline
\end{tabular}

\subsection{La ejecución de la medida}

Una vez analizadas las características psicosociales de los menores de la muestra, así como de su historial delictivo y de las reacciones al mismo, es preciso dar cuenta de la ejecución de la Libertad Vigilada: de los objetivos que aspira a conseguir, de las intervenciones propuestas y de los recursos con que se cuenta. Sobre todo para, en un segundo momento, poder valorar el éxito de la medida en función de los cambios experimentados en los menores.

\subsection{1. ¿Qué se hace con el menor? Objetivos, intervenciones y recursos}

El PIEM de cada menor es elaborado por el técnico encargado de ejecutar la medida de Libertad Vigilada. En él se especifican una serie de objetivos, intervenciones y tareas que el menor ha de llevar a cabo durante la realización de la medida. Estos extremos se concretan teniendo en cuenta, tanto el contenido de la sentencia, donde el juez ha podido imponer una tarea particular, como las circunstancias sociales y personales de cada menor. Teniendo en cuenta que la Libertad Vigilada es una medida que permite una enorme individualización, el PIEM tratará de incidir en las circunstancias más deficitarias del menor, apoyándose en sus potencialidades ${ }^{5}$; para conseguir finalmente la responsabilización y reinserción del menor.

En ese sentido, de alentar la individualización de la medida, el artículo 18 del RD 1774/2004, establece que el técnico realizará una valoración de las siguientes áreas: conflicto (conducta antisocial y delictiva), desarrollo individual (área psicológica),

\footnotetext{
${ }^{5}$ Una información más amplia sobre este aspecto y sobre el procedimiento y las cuestiones que se tienen en cuenta en la elaboración del PIEM se puede ver en Bernuz, Fernández, Pérez, 2009.
}

Revista Española de Investigación Criminológica

Artículo 6, Número 7 (2009) $\quad$ www.criminología.net

ISSN: $1696-9219$ 
salud, familiar, relacional y social (conflictos, expectativas y recursos de los que se dispone en el entorno del menor) y formativo laboral. Una vez valoradas todas las áreas, el técnico deberá proponer actividades concretas que le permitan programar la tarea de acompañamiento o seguimiento del menor, su supervisión y control, así como mantener la motivación en los distintos ámbitos. Hay que tener en cuenta, como indican los propios educadores, que quizá los fines educativos y las expectativas de cambio son desmesuradas atendiendo al tiempo de intervención real con que se cuenta. En la muestra, el 43,3\% de las sentencias condenan a seis meses de Libertad Vigilada y el $48,4 \%$ a un año.

a) Objetivos planteados e intervenciones propuestas

En cuanto a los objetivos a lograr con la ejecución de la medida, hay que destacar que aquéllos en los que más se insiste en esta muestra son los relativos a la asunción de responsabilidad y mejora de comportamiento y los que inciden en las actividades formativas y/o laborales, que aparecen reflejados en la casi unanimidad de los expedientes ${ }^{6}$; seguidos de un control de consumo de tóxicos, de amistades y, en último lugar, están los objetivos relacionados con el ámbito familiar (tabla 7).

Tabla 7. Objetivos marcados en los PIEM

\begin{tabular}{|l|c|c|}
\hline & $\mathbf{n}$ & $\mathbf{\%}$ \\
\hline Objetivos comportamiento/responsabilidad & 58 & 96,7 \\
\hline Objetivos formativos/laborales & 58 & 96,7 \\
\hline Objetivos tóxicos/salud & 39 & 65 \\
\hline Objetivos entorno/iguales & 29 & 48,3 \\
\hline Objetivos familiares & 22 & 36,7 \\
\hline
\end{tabular}

Si nos detenemos en las intervenciones concretas propuestas a los menores, se puede obtener una información más diferenciada. Así, entre las tareas que habitualmente se proponen están, de un lado, las relativas a los procesos de búsqueda de empleo: acudiendo a actividades de orientación laboral o a la oficina de desempleo; o relativas al mantenimiento del puesto de trabajo. De otro lado, se propone la realización de talleres que apoyen aspectos deficitarios del comportamiento del menor: habilidades sociales, educación sexual, control de impulsos y similares. En ese sentido, la tabla 8, refleja la diversidad de tareas que se incluyen en los PIEM.

\footnotetext{
${ }^{6}$ Sobre la importancia del objetivo de la formación o la inserción familiar, se pueden ver las consideraciones de los educadores en Bernuz, Fernández y Pérez, 2009.
}

Revista Española de Investigación Criminológica

Artículo 6, Número 7 (2009) $\quad$ www.criminología.net

ISSN: $1696-9219$ 
Tabla 8. Intervenciones propuestas en los PIEM

\begin{tabular}{|l|c|c|}
\hline & $\mathbf{n}$ & $\mathbf{\%}$ \\
\hline Promoción empleo, búsqueda, continuación & 41 & 68,3 \\
\hline Talleres habilidades sociales, drogas, sexualidad & 37 & 61,7 \\
\hline Control tóxicos o prevención & 29 & 48,3 \\
\hline Actividades formativas, refuerzo estudios & 25 & 41,7 \\
\hline Comportamiento adecuado en casa & 22 & 36,7 \\
\hline Entrevistas familiares, tratamiento familiar & 22 & 36,7 \\
\hline Evitar compañías antisociales & 17 & 28,3 \\
\hline Actividades tiempo libre & 14 & 23,3 \\
\hline Tratamiento psicológico o médico & 12 & 20 \\
\hline No cometer delitos & 11 & 18,3 \\
\hline Reflexión delito & 10 & 16,7 \\
\hline Apoyo educativo o de comportamiento & 9 & 15 \\
\hline $\begin{array}{c}\text { Comportamiento adecuado en colegio, trabajo, } \\
\text { talleres }\end{array}$ & 8 & 13,3 \\
\hline $\begin{array}{l}\text { Comportamiento adecuado con educador, acudir } \\
\text { citas }\end{array}$ & 6 & 10 \\
\hline
\end{tabular}

Uno de los elementos centrales de este análisis es el estudio de la adecuación de las tareas propuestas a las circunstancias psicosociales del menor, atendiendo a la individualización requerida en la justicia de menores. Para ello se ha realizado un análisis de significación entre las distintas problemáticas y carencias de los menores y las intervenciones propuestas para mejorar esas situaciones. En todos los casos los resultados dejan ver que no se diferencian las tareas propuestas entre los menores que sufren o no una deficiencia concreta. La única excepción es el caso de los jóvenes que tienen problemas con las drogas, en cuyo caso sí se propone un tratamiento diferenciado y específico para hacer desaparecer su toxicomanía, como se comprueba en la tabla 9.

Tabla 9. Intervención propuesta para los menores con problemas de drogadicción*

\begin{tabular}{|l|l|c|c|c|}
\hline \multicolumn{2}{|c|}{} & \multicolumn{2}{|c|}{ Problemas con drogas } & Prueba de significación \\
\cline { 3 - 4 } \multicolumn{2}{|c|}{} & $\begin{array}{c}\text { No } \\
\mathrm{n} \%\end{array}$ & $\begin{array}{c}\text { Sí } \\
\mathrm{n} \%\end{array}$ & \multirow{2}{*}{$\%$} \\
\multirow{2}{*}{ Tarea de control de tóxicos } & Sí & $5(27,8)$ & $23(69,7)$ & \multirow{2}{*}{$\chi^{2}=8,266 ;$ g.1.=1; $\mathrm{p} \leq 0,01$} \\
\cline { 2 - 4 } & No & $13(72,2)$ & $10(30,3)$ & \\
\hline
\end{tabular}

* Calculado para una tabla de $2 \times 2$

Para conocer cómo se han asignado las tareas a los distintos casos se ha comprobado qué relación existe entre la asignación de dicha tarea y las variables que proporcionan la información psicosocial del menor. Así por ejemplo, las tareas de evitar compañías antisociales y fomentar actividades de tiempo libre se han puesto en relación

Revista Española de Investigación Criminológica

Artículo 6, Número 7 (2009) $\quad$ www.criminología.net

ISSN: $1696-9219$ 
con la variable "tener amigos problemáticos". Los resultados, en todos los casos, indican que hay menores que tienen un déficit en un aspecto psicosocial concreto $\mathrm{y}$, sin embargo, no se les propone una intervención que la pueda subsanar. También se observa otra paradoja, la de aquellos menores a los que se les impone una tarea determinada a pesar de que en el PIEM no se recoge que el menor tiene un déficit en esa área. En las siguientes tablas 10 y 11 se muestran dos ejemplos ilustrativos.

Tabla 10. Intervención para los menores con amigos problemáticos

\begin{tabular}{|l|l|c|c|}
\hline \multirow{2}{*}{} & & \multicolumn{2}{|c|}{ Amigos problemáticos } \\
\cline { 3 - 4 } & & \multicolumn{2}{c|}{$\begin{array}{c}\text { No } \\
\text { Sí }\end{array}$} \\
\hline \multirow{2}{*}{ Evitar compañías antisociales } & Sí & $2(13,3)$ & $13(34,2)$ \\
\cline { 2 - 4 } & No & $13(86,7)$ & $25(65,8)$ \\
\cline { 2 - 4 } & Total & $15(100)$ & $38(100)$ \\
\hline
\end{tabular}

Tabla 11. Intervención para los menores con problemática escolar

\begin{tabular}{|l|l|c|c|}
\hline \multirow{2}{*}{} & \multicolumn{2}{|c|}{\begin{tabular}{c} 
Problemática escolar \\
No \\
\cline { 3 - 4 }
\end{tabular}} & $\begin{array}{c}\text { Só } \\
\mathrm{n} \%\end{array}$ \\
\hline \multirow{2}{*}{ Actividades formativas } & Sí & $8(61,5)$ & $17(36,2)$ \\
\cline { 2 - 4 } & No & $5(38,5)$ & $30(63,8)$ \\
\cline { 2 - 4 } & Total & $13(100)$ & $47(100)$ \\
\hline
\end{tabular}

b) Recursos empleados

Una vez analizados los objetivos y las intervenciones propuestas con los menores de la muestra, es preciso conocer los recursos a los que se acude para cubrir la amplia gama de necesidades educativas del menor. Hay que tener en cuenta que el menor no siempre tiene que realizar actividades adicionales y el PIEM únicamente incide en la necesidad de que el menor continúe con las obligaciones familiares, formativas y/o laborales que ya viene desarrollando habitualmente.

Igualmente, es preciso destacar que los educadores van a acudir, tanto a sus propios recursos, como a otros externos. Así, en algunas ocasiones, es alguno de los educadores del Equipo de Medio Abierto el que ofrece un taller concreto o la formación específica para que el menor, o un grupo de menores con similares carencias, cumplan con la tarea formativa impuesta; generalmente en el ámbito de las habilidades sociales o de las pautas de comportamiento. No obstante, lo más usual es que, para cumplir con los

Revista Española de Investigación Criminológica 
objetivos de su medida, el menor tenga que acudir a uno o varios servicios externos que podrán ser tanto públicos, como privados. Agrupados por temáticas podríamos decir que son tres los tipos de recursos más utilizados: los de salud (centros de salud, centros de deshabituación de drogodependencias, unidades de salud mental, etc), los formativos (Institutos de ESO, otros centros de formación reglada, etc.) y los laborales (INEM, programas públicos y privados de inserción laboral, etc.).

En líneas generales, los técnicos consideran que tienen suficientes recursos. Ahora bien, hay sectores de actuación que están menos dotados o pueden mejorar sus prestaciones; así los recursos de salud mental y de apoyo psicológico para jóvenes suelen ser insuficientes y/o faltarles agilidad en su respuesta. Lo mismo sucede con los servicios que ofrecen terapia y control de drogodependencias. También son escasos los recursos que ofrecen apoyo multidisciplinar a las familias con carencias graves y globales. En ocasiones, la burocracia y las incompatibilidades en la gestión pública por intransigencia de las diversas administraciones provocan que los técnicos se encuentren con problemas de acceso a los recursos. No obstante, la experiencia acumulada y el conocimiento mutuo entre los profesionales de diversos ámbitos, suelen contrarrestar dichas dificultades.

\subsection{2. ¿Qué se consigue? Objetivos conseguidos, incumplimiento, cambios, pronósticos}

En este apartado se va a tratar de analizar la eficacia de la ejecución de la medida. Para ello se van a analizar los resultados obtenidos, tanto a través de los objetivos conseguidos, como de los cambios que se observan en el menor al finalizar la ejecución de la medida. Eso sí, siempre desde la perspectiva de los educadores, recogida en los expedientes y manifestada en las entrevistas.

a) Objetivos conseguidos

En primer lugar habría que poner de manifiesto que el 83\% de los menores asisten de manera habitual a las entrevistas (esto es, a más de un $75 \%^{7}$ ). Y el $78 \%$ también lo hace así con el resto de las actividades. Y es que, en general, los menores cumplen con

\footnotetext{
${ }^{7}$ Ver explicación en página 5.
} 
el plan de trabajo establecido en el PIEM. Según los resultados obtenidos, un 63,5\% cumple siempre y un $25 \%$ cumple casi siempre.

Tabla 12. Correlación entre grado de consecución de objetivos y desarrollo del PIEM

\begin{tabular}{|c|c|c|c|c|c|c|}
\hline & & $\begin{array}{l}\text { Asistencia } \\
\quad \text { a las } \\
\text { entrevistas }\end{array}$ & $\begin{array}{l}\text { Asistencia } \\
\text { a las } \\
\text { actividades }\end{array}$ & $\begin{array}{l}\text { Cumplimiento } \\
\text { del plan de } \\
\text { trabajo }\end{array}$ & $\begin{array}{l}\text { Implicación } \\
\text { del menor }\end{array}$ & $\begin{array}{c}\text { Grado de } \\
\text { consecución } \\
\text { de objetivos }\end{array}$ \\
\hline \multirow[t]{3}{*}{$\begin{array}{l}\text { Asistencia a } \\
\text { las entrevistas }\end{array}$} & $\begin{array}{l}\text { Correlación } \\
\text { de Pearson }\end{array}$ & 1 &, $704(* *)$ &, $652(* *)$ &, $718(* *)$ &, $626(* *)$ \\
\hline & $\begin{array}{l}\text { Sig. } \\
\text { (bilateral) }\end{array}$ & &, 000 &, 000 &, 000 &, 000 \\
\hline & $\mathrm{N}$ & 53 & 52 & 52 & 53 & 53 \\
\hline \multirow{3}{*}{$\begin{array}{l}\text { Asistencia a } \\
\text { las } \\
\text { actividades }\end{array}$} & $\begin{array}{l}\text { Correlación } \\
\text { de Pearson }\end{array}$ & & 1 &, $763(* *)$ &, $763(* *)$ &, $580(* *)$ \\
\hline & $\begin{array}{l}\text { Sig. } \\
\text { (bilateral) }\end{array}$ & & &, 000 &, 000 & ,000 \\
\hline & $\mathrm{N}$ & & 52 & 51 & 52 & 52 \\
\hline \multirow{3}{*}{$\begin{array}{l}\text { Cumplimiento } \\
\text { del plan de } \\
\text { trabajo }\end{array}$} & $\begin{array}{l}\text { Correlación } \\
\text { de Pearson }\end{array}$ & & & 1 &, $717(* *)$ &, $574(* *)$ \\
\hline & $\begin{array}{l}\text { Sig. } \\
\text { (bilateral) }\end{array}$ & & & &, 000 &, 000 \\
\hline & $\mathrm{N}$ & & & 52 & 52 & 52 \\
\hline \multirow[t]{3}{*}{$\begin{array}{l}\text { Implicación } \\
\text { del menor }\end{array}$} & $\begin{array}{l}\text { Correlación } \\
\text { de Pearson }\end{array}$ & & & & 1 &, $756(* *)$ \\
\hline & $\begin{array}{l}\text { Sig. } \\
\text { (bilateral) }\end{array}$ & & & & &, 000 \\
\hline & $\mathrm{N}$ & & & & 60 & 60 \\
\hline \multirow{3}{*}{$\begin{array}{l}\text { Grado de } \\
\text { consecución } \\
\text { de objetivos }\end{array}$} & $\begin{array}{l}\text { Correlación } \\
\text { de Pearson }\end{array}$ & & & & & 1 \\
\hline & $\begin{array}{l}\text { Sig. } \\
\text { (bilateral) }\end{array}$ & & & & & \\
\hline & $\mathrm{N}$ & & & & & 60 \\
\hline
\end{tabular}

En principio, tal y como muestran los resultados de la tabla 12 existe una correlación estadísticamente significativa, entre la observancia de los objetivos y la asistencia habitual a entrevistas y actividades, con el correcto cumplimiento del plan de trabajo y con la buena implicación de los menores; y viceversa, no cumplir con los objetivos y no asistir con frecuencia a las entrevistas y actividades, están en relación con el incumplimiento del plan de trabajo y la mala implicación del menor en el plan de Libertad Vigilada. Sin embargo, destaca la existencia de un pequeño grupo de menores que a pesar de asistir o cumplir con lo previsto, no logran que el cumplimiento de la medida haya desplegado los efectos deseados. En efecto, tal y como revelan los resultados del análisis, la consecución plena de los objetivos previstos sólo se ha

Revista Española de Investigación Criminológica

Artículo 6, Número 7 (2009) $\quad$ www.criminología.net

ISSN: $1696-9219$ 
obtenido con un $46,7 \%$ de los menores. Por otra parte, si se comprueban los resultados de la tabla 12 parece que la consecución de los objetivos se relaciona en mayor medida con una buena implicación del menor y en menor medida con el cumplimiento de plan de trabajo.

Todos estos resultados coinciden con la valoración personal que los distintos profesionales hicieron de cada uno de los casos; así, según estos, en un 63,3\% de las medidas ejecutadas los técnicos hicieron una valoración favorable o muy favorable de lo que había sido el desarrollo de la medida.

b) Cambios en el menor

Para analizar la eficacia de la Libertad Vigilada, además de estudiar los objetivos conseguidos, se consideró importante determinar si el cumplimiento de la medida ha supuesto cambios positivos en la vida del menor. La valoración de estos cambios se hizo en relación a las cinco áreas de actuación que los profesionales suelen abordar de manera independiente: familiar, formativo/laboral, grupo de pares, aspectos personales (psicológicos y adictivos) y responsabilización.

Tabla 13. Relación entre las áreas de intervención y los cambios obtenidos

\begin{tabular}{|l|c|c|c|c|c|}
\hline & $\begin{array}{c}\text { Cambio } \\
\text { positivo }\end{array}$ & $\begin{array}{c}\text { Sin cambio } \\
\text { o cambio } \\
\text { negativo }\end{array}$ & $\begin{array}{c}\text { Sin } \\
\text { necesidad } \\
\text { de cambio }\end{array}$ & No consta & Total \\
\cline { 2 - 6 } & $\mathrm{n}(\%)$ & $\mathrm{n}(\%)$ & $\mathrm{n}(\%)$ & $\mathrm{n}(\%)$ & $\mathrm{N}(\%)$ \\
\hline Área de familia & $32(53,3)$ & $22(36,7)$ & $5(8,3)$ & $1(1,7)$ & $60(100)$ \\
\hline Área formativo laboral & $34(56,7)$ & $16(26,7)$ & $3(5)$ & $7(11,7)$ & $60(100)$ \\
\hline Área grupo de pares & $18(30)$ & $12(20)$ & $14(23,3)$ & $16(26,7)$ & $60(100)$ \\
\hline Área personal & $19(31,7)$ & $19(31,7)$ & $20(33,3)$ & $2(3,3)$ & $60(100)$ \\
\hline $\begin{array}{l}\text { Área de } \\
\text { responsabilización }\end{array}$ & $39(65)$ & $19(31,7)$ & 0 & $2(3,3)$ & $60(100)$ \\
\hline
\end{tabular}

Como puede observarse en la tabla 13, en la penúltima columna, el número de no consta es muy elevado en algunas áreas, especialmente en la del grupo de pares, en la medida que en algunos de los informes finales, algunas áreas quedaron sin valorar. Salvando este aspecto, y manejando sólo la información de la que se dispone, para valorar la eficacia de la intervención no sólo habrá que analizar los cambios positivos obtenidos sino también la información que muestra la tercera columna, que determina 
en cada caso si esa área se estableció como objetivo del PIEM y la información que muestra la segunda columna, que indica la imposibilidad de la medida para proporcionar cambios en la vida del menor. Así combinando la información de todas las columnas, se puede comprobar que las áreas de responsabilización y la formativo/laboral, que son áreas prioritarias en la intervención desde la medida de Libertad Vigilada, muestran el mejor balance. Por su parte, en el área familiar, aunque se obtienen muchos cambios positivos, hay también muchos fracasos.

Finalmente, el área personal (adicciones y déficits psicológicos) y la del grupo de pares, se muestran como ámbitos dónde se presentan mayores dificultades y fracasos en la intervención. En este sentido, y a juzgar por los resultados del análisis, parece que la problemática de la que se parte es difícil de modificar a través de la ejecución de esta medida (ver tabla 14). Así, sólo en estas dos áreas se observan diferencias estadísticamente significativas entre la situación psicosocial de la que parte el menor y los cambios conseguidos. Esto es, la existencia de un problema de salud mental, de consumos o de amigos problemáticos, parece ser un impedimento para obtener cambios positivos a través de la intervención.

Tabla 14. Cambios con el grupo de pares e individuales conseguidos en relación con los problemas psicosociales de partida

\begin{tabular}{|c|c|c|c|c|c|c|}
\hline \multicolumn{7}{|c|}{ No } \\
\hline & & $\begin{array}{c}\text { Cambio } \\
\text { positivo } \\
\text { n (\%) }\end{array}$ & $\begin{array}{c}\text { No } \\
\text { cambio o } \\
\text { negativo } \\
n(\%)\end{array}$ & $\begin{array}{c}\text { No } \\
\text { necesidad } \\
\text { n (\%) }\end{array}$ & $\begin{array}{l}\text { No consta } \\
\mathrm{n}(\%)\end{array}$ & Prueba de significación \\
\hline \multirow{2}{*}{$\begin{array}{l}\text { Salud } \\
\text { mental }\end{array}$} & Sí & $8(40)$ & $10(50)$ & 0 & $2(10)$ & \multirow{2}{*}{$\chi^{2}=14,145 ; g .1=3 ; p \leq 0,01$} \\
\hline & No & $6(26,1)$ & $6(26,1)$ & $11(47,8)$ & 0 & \\
\hline \multirow{2}{*}{$\begin{array}{l}\text { Consumo } \\
\text { de drogas }\end{array}$} & Sí & $8(24,2)$ & $16(48,5)$ & $8(24,2)$ & $1(3)$ & \multirow{2}{*}{$\chi^{2}=8,185 ;$ g.l. $=3 ; p \leq 0,05$} \\
\hline & No & $8(44,4)$ & $2(11,1)$ & $8(44,4)$ & 0 & \\
\hline \multirow{2}{*}{$\begin{array}{l}\text { Consumo } \\
\text { de alcohol }\end{array}$} & Sí & $5(26,3)$ & $11(57,9)$ & $3(15,8)$ & 0 & \multirow{2}{*}{$\chi^{2}=7,287 ;$ g.1. $=2 ; p \leq 0,05$} \\
\hline & No & $7(33,3)$ & $4(19)$ & $10(76,9)$ & 0 & \\
\hline \multirow{2}{*}{$\begin{array}{l}\text { Amigos } \\
\text { conflictivos }\end{array}$} & Sí & $18(47,4)$ & $9(23,7)$ & 0 & $11(28,9)$ & \multirow{2}{*}{$\chi^{2}=44,047 ;$ g.l. $=3 ; p \leq 0,01$} \\
\hline & No & 0 & $1(6,7)$ & $13(86,7)$ & $1(6,7)$ & \\
\hline
\end{tabular}

\section{Discusión}

Los datos muestran muchas cuestiones sobre las que discutir que nos permiten llegar a algunas conclusiones sobre cómo se materializa en la práctica la ejecución de la 
medida de Libertad Vigilada y, sobre todo, sobre su capacidad efectiva para individualizar la medida judicial y fomentar cambios en el menor.

Así, por ejemplo, llama la atención que la inmensa mayoría de los delitos cometidos por los menores de la muestra $(68,3 \%)$ son delitos que, al margen de la gravedad, han sido cometidos con violencia e intimidación. De manera que se podría pensar que con esta medida se trabaja fundamentalmente el rasgo violento de los delitos, al margen de su calificación penal como grave o no. Quizás porque se parte de la idea de que lo que indica una mayor peligrosidad en los menores es la comisión del delito o falta con violencia. Y se considera preciso trabajar con carácter primordial sobre esa cuestión.

Resulta interesante también comprobar cómo los menores que cumplen una medida de Libertad Vigilada se adaptan al perfil del menor 'tipo' de la justicia de menores, esto es, perteneciente a una familia desestructurada, con una dinámica de educación inadecuada, que ha tenido contacto con los Servicios Sociales -aunque en un $50 \%$ de los casos no eran menores conocidos por los mismos-, con un nivel de escolarización muy bajo y en estos casos con problemas de conducta, que se relaciona con amigos problemáticos y que tiene problemas con las drogas. Problemas que, según el concepto que se maneje de la Protección de la Infancia, formarían parte de sus competencias según la LO 1/1996, de protección jurídica del menor. Quizás se podría destacar que la Libertad Vigilada, en tanto es la medida más tradicional de la justicia de menores, sigue funcionando para un menor 'tipo': el que se ha encontrado fundamentalmente en la muestra. Puede que para los menores que han cometido delitos más leves o que pertenecen a un contexto social y familiar normalizado se estén aplicando otras medidas más suaves de la justicia de menores.

No obstante, también hay que destacar que de los menores de la muestra, algo menos de la mitad $(43,4 \%)$ no tenían antecedentes previos en el juzgado, por lo que la Libertad Vigilada analizada era la primera sanción judicial que recibían. Resulta curioso que esta medida, que según la legislación es una de las más restrictivas de derechos, sea la primera en imponerse desde la jurisdicción de menores. Y no, como en principio debería ser, esto es, que se aplique una vez se ha evidenciado el fracaso de otras medidas no privativas de libertad. En sentido contrario, también resulta curioso que algunos menores de la muestra, antes de cumplir esa Libertad Vigilada, ya han

Revista Española de Investigación Criminológica 
cumplido previamente algún tipo de internamiento. En este caso el sistema estaría abandonando otro de los principios más consolidados de la justicia de menores que propugna toda la normativa internacional: a la hora de enjuiciar al menor se aplica cualquiera de las medidas propuestas legalmente, pero de forma progresiva, empezando en las primeras intervenciones con las medidas que inciden fundamentalmente en la educación y la responsabilización del menor, y avanzando hacia aquéllas en las que predomina el componente de control, si el menor continúa con sus conductas delictivas.

Por lo general, operadores jurídicos y sociales parecen afirmar que la Libertad Vigilada es la medida de medio abierto que permite una mayor y mejor individualización de la respuesta atendiendo a las circunstancias psicosociales de los menores. Sin embargo, si se atiende a los resultados obtenidos en la investigación, se puede comprobar que, en ocasiones, determinadas carencias no van asociadas a unas exigencias en el PIEM. $\mathrm{O}$ al contrario, que hay actividades que no se relacionan con carencias del menor. Tan sólo, la existencia de problemas con consumos de alcohol y/o drogas son los que están directamente relacionados con la concreción de un objetivo en esta área.

Además, para lograr la individualización de la respuesta también es importante que ésta se imponga y ejecute lo más rápidamente posible para alentar el proceso de responsabilización del menor, y para que el menor relacione la medida impuesta con el delito cometido. Según la muestra estudiada, los menores comienzan a delinquir en el margen del ámbito subjetivo de la justicia de menores (14,65 años), el delito por el que se les impone la Libertad Vigilada lo cometen un año después y se empieza a ejecutar poco antes de llegar a la mayoría de edad penal (17,4 años). Es evidente que debe transcurrir todo el procedimiento para que se pueda imponer una Libertad Vigilada, o que a veces se abre una distancia entre el delito y la respuesta porque hay otras medidas - de internamiento - que deben cumplirse con prioridad. No obstante, también hay que ser conscientes de que un lapso de tiempo tan amplio podría hacer ineficaz cualquier medida -incluso la de Libertad Vigilada.

Es posible que detrás de esas lagunas o contradicciones exista una cierta rutina en la forma de elaborar los propios PIEM que se centran fundamentalmente y conceden una importancia mayor a los objetivos relacionados con la responsabilización del menor y con el ámbito formativo-laboral en todo caso; dejando más de lado los objetivos que

Revista Española de Investigación Criminológica 
tienen que ver con la familia, los amigos o los propios problemas personales del menor, a excepción del consumo abusivo de drogas que si parece ser un objetivo prioritario. Quizás porque se considera que los dos objetivos citados son los fundamentales en una edad en la que se pretende la autonomía del menor, o porque consideran que la integración formativa y laboral conseguirá afianzar por sí sola el resto de los objetivos (Bernuz, Fernández, Pérez, 2009). En todo caso, los propios educadores también se han preocupado de poner de manifiesto que en el tiempo que dura la Libertad Vigilada (generalmente inferior a un año) no se pueden conseguir objetivos a largo plazo.

En cuanto a los recursos para ejecutar la medida de Libertad Vigilada, lo cierto es que todos los educadores parecen estar de acuerdo en que, en general, hay suficientes recursos para trabajar con los menores, y no se concede excesiva importancia a si son recursos privados o públicos.

Finalmente, en relación con el grado de eficacia de la medida, aspecto crucial de cara a la legitimación de la justicia de menores ante la sociedad y frente al propio menor implicado, se podría afirmar que los resultados que se obtienen tras la ejecución de la medida, parecen en líneas generales positivos. Así, sólo consta el incumplimiento en un $13,3 \%$ de los casos y la reincidencia posterior a la ejecución de la medida se sitúa en un $21,7 \%{ }^{8}$. No obstante, aparte de la reincidencia, existen otros indicadores que nos permiten evaluar la eficacia de la medida. Así, profundizando en el desarrollo global de la medida, se ha podido comprobar que en un $46,7 \%$ de casos se ha conseguido la consecución plena de los objetivos. Y que este resultado se relaciona más con una buena implicación del menor y no tanto con el cumplimiento global del PIEM. En efecto, los técnicos, en su informe final para el Juez, han valorado de manera favorable o muy favorable una mayor cantidad de casos, un $63,3 \%$. Por ello podría decirse que, aunque no se hayan conseguido exactamente todos los objetivos propuestos al inicio de la medida, parece que los técnicos valoran los cambios positivos que va realizando el menor y que demuestran una mejora de su responsabilidad y su implicación. De hecho, como se comentaba anteriormente, las áreas prioritarias de intervención se centran en dos: responsabilización y formativo/laboral, y éstas son en las que se consiguen

\footnotetext{
${ }^{8}$ Esta tasa de reincidencia también se obtiene en otros estudios, así, la reciente investigación llevada a cabo en el País Vasco informa que la tasa de reincidencia de los menores que han finalizado una medida de medio abierto es del 21,7\%. Cfr. San Juan y Ocáriz (2009). Pg. 69
}

Revista Española de Investigación Criminológica

Artículo 6, Número 7 (2009) $\quad$ www.criminología.net

ISSN: $1696-9219$ 
mayores cambios; mientras que en las áreas personal (salud mental y consumos) y de ocio se observan más dificultades para conseguir alguna mejoría.

\section{Conclusiones}

En síntesis, este estudio nos ofrece algunas razones para entender por qué la Libertad Vigilada fue y sigue siendo una medida fundamental en la justicia de menores española, a pesar de las dificultades y contradicciones que su ejecución conlleva y que se han puesto de manifiesto. Y lo es, tanto por el sujeto con el que se interviene, como por el tipo de intervención que se propone. Los menores con los que se interviene conforman una población 'típica' de la jurisdicción juvenil más tradicional, esto es, un niño o adolescente con unas circunstancias personales, familiares, escolares y sociales complejas que exijen un tratamiento individualizado. El propio contenido de la medida permite en principio la adaptación a la mayoría de las circunstancias del menor y -en menor medida - a su evolución a lo largo de la ejecución de la misma; esto es, hace posible la individualización de la medida que evita que ésta se limite a ser proporcional al hecho cometido. Lo cual demuestra que, efectivamente, la justicia de menores es un ámbito donde la respuesta al delito obedece a criterios "especiales" y no por ello se considera que deje de ser efectiva.

Ahora bien, también se ha constatado una cierta homogeneidad en la práctica que, en ocasiones, hace que las intervenciones se parezcan mucho entre unos casos y otros-, que nos permite concluir que la individualización puede ser más teórica que real. Esta homogeneidad puede ser debida a varios factores. A veces puede ocurrir que, como muestran los datos del estudio, los menores a los que se les impone la medida cuentan todos ellos con un perfil bastante parecido y por lo tanto sus necesidades también son similares. También el hecho de que los recursos disponibles sean limitados puede condicionar una reiteración en las intervenciones. Finalmente, no hay que olvidar que, a pesar de que la Libertad Vigilada puede ser una buena oportunidad para intervenir con el menor y mejorar algunas de las carencias que se han detectado en su entorno psicosocial, se trata de una intervención puntual y no muy extensa en el tiempo. Razón por la cual el educador puede preferir centrarse en aquellos aspectos más dinámicos que son modificables más fácilmente (por ejemplo, el ámbito laboral) y dejar en una segunda posición objetivos más difíciles de abordar de manera esporádica,

Revista Española de Investigación Criminológica

Artículo 6, Número 7 (2009) $\quad$ www.criminología.net

ISSN: $1696-9219$ 
esperando que la consecución de aquéllos permitan de manera tangencial mejorar otros aspectos.

En definitiva, se puede concluir que efectivamente y a pesar de las dificultades que pueden surgir durante la ejecución y que se han puesto de manifiesto en los resultados, esta medida es una medida óptima para trabajar con menores que cuentan con perfiles sociales complejos y que han cometido delitos en los que ha mediado algún tipo de violencia. Y, en consecuencia, hace que podamos augurar que seguirá siendo una medida central en la justicia de menores española. El reto para el futuro es, además de mejorar las deficiencias comentadas, idear nuevas estrategias de control, de educación y de reinserción que permitan que esta medida se pueda poner en marcha con menores que cometan también delitos graves y con los menores más conflictivos para que, en efecto, se convierta en una alternativa al internamiento.

\section{Bibliografía}

BERNUZ BENEÍTEZ, M‥J. (1999): De la protección de la infancia a la prevención de la delincuencia. Zaragoza: Colección Justicia de Aragón.

BERNUZ BENEITEZ, Ma J., FERNÁNDEZ MOLINA, E., PÉREZ JIMÉNEZ, F. (2009): Educar y controlar: un análisis en profundidad de la ejecución de la Libertad Vigilada en la justicia de menores. Enviado para su publicación a la Revista Electrónica de Ciencia Penal y Criminología. Pendiente de revisión.

CAPDEVILLA CAPDEVILLA, M., FERRER PUIG, M., LUQUE REINA, E. (2005): La reincidencia en el delito en la justicia de menores. Documentos de Trabajo. Barcelona, Centre d'Estudis Juridics i Formació Especialitzada.

FERNÁNDEZ MOLINA, E. (2008): Entre la educación y el castigo: un análisis de la justicia de menores en España. Valencia: Tirant lo Blanch.

GRAÑA GÓMEZ, J.L., GARRIDO GENOVÉS, V., GONZÁLEZ CIEZA, L. (2007): Reincidencia delictiva en menores infractores de la comunidad de Madrid: evaluación, características delictivas y modelos de predicción. Madrid, Agencia para la reeducación y reinserción del menor infractor.

GARCIA PÉREZ, O. (Dir.), DÍEZ RIPOLLÉS, J.L., PÉREZ JIMÉNEZ, F., GARCÍA RUIZ, S. (2008): La delincuencia juvenil ante los Juzgados de Menores. Valencia: Tirant lo Blanch.

IASS (2009): La intervención con los menores de edad en conflicto con la ley penal. Proyecto educativo del área de Atención al Menor en conflicto social, Zaragoza, IASS.

PEREZ JIMÉNEZ, F. (2006): Menores infractores: Estudio empírico de la respuesta penal. Valencia: Tirant lo Blanch.

Revista Española de Investigación Criminológica

Artículo 6, Número 7 (2009) $\quad$ www.criminología.net

ISSN: $1696-9219$ 
RECHEA ALBEROLA, C., FERNÁNDEZ MOLINA, E. (2001): La nueva Justicia de Menores. Cuadernos de Política Criminal 74, pp. 325-353.

SAN JUÁN GUILLÉN, C., OCÁRIZ PASSEVANT, E. (2009): Evaluación de la intervención educativa y análisis de la reincidencia en la Justicia de Menores en la $\boldsymbol{C A P V}$. Vitoria-Gasteiz: Gobierno Vasco. 